\title{
PLURALISM AND PROOFS
}

\author{
Greg Restall* \\ Philosophy Department, \\ The University of Melbourne
}

restall@unimelb.edu.au

August 2011

\begin{abstract}
Beall and Restall's Logical Pluralism [2] characterises pluralism about logical consequence in terms of the different ways cases can be selected in the analysis of logical consequence as preservation of truth over a class of cases. This is not the only way to understand or to motivate pluralism about logical consequence. Here, I will examine pluralism about logical consequence in terms of different standards of proof. We will focus on sequent derivations for classical logic, imposing two different restrictions on classical derivations to produce derivations for intuitionistic logic and for dual intuitionistic logic. The result is another way to understand the manner in which we can have different consequence relations in the one language. Furthermore, the proof-theoretic perspective gives us a different explanation of how the one concept of negation can have three different truth conditions, those in classical, intuitionistic and dual-intuitionistic models.
\end{abstract}

I am a pluralist about logical consequence [1, 2]. From the beginning of my interest in pluralism about logical consequence, my motivation has remained unchanged: I take it that there are particular, concrete arguments, for which the question 'is this argument valid?' has more than one correct and informative answer. My first example was arguments of the form of disjunctive syllogism (from $p \vee q$ and $\neg p$ to $q$ ) and explosion (from $p$ and $\neg p$ to $q$ ). In both of these cases, I became convinced that the most appropriate answer was twofold:

*See http://consequently.org/writing/pluralism_and_proofs/for the latest version of the paper, to post comments and to read comments left by others. T Thanks to audiences at the "Logical Pluralism" workshop in Tartu, the Logic Seminar at the University of Melbourne, and the Logic or Logics Workshop of the Foundations of Logical Consequence Project at Arché at the University of St Andrews for comments on this paper. I I was supported by the ARC Discovery Grants DPO556827 and DP1094962, and Tonio K's Life in the Foodchain. 
yes, the argument is valid-in the sense that it is impossible for the premises to be true and the conclusion to be false-and no, the argument is invalid, for there are impossible circumstances (circumstances inconsistent about $p$, but in which q fails) in which the premises hold and the conclusion doesn't. The one and the same argument can be both valid in one sense and invalid in the other. It is not that the meaning of negation changes between one argument and the other: the negation is understood in the one and the same way across both arguments. If there were equivocation between two senses of negation, then either in the first case it need not be impossible for the premises to be true, or in the second case they need not be impossible circumstances in which $p$ and $\neg p$ are both true. I came to agree that both a kind of classical consequence in which inconsistent premises entail every conclusion, and a stronger notion of consequence, in which inconsistent premises need not entail every conclusion, for inconsistencies - though still inconsistent-are allowed as true at some 'points' in an interpretation. An interpretation may treat some things inconsistently without treating other things inconsistently. We may be inconsistent about $p$ (double-mindedly agreeing both with $p$ and with $\neg p$ ) without thereby being inconsistent about $\mathrm{q}^{\top}$

This motivation for relevant consequence, and the concomitant sense in which a mistake is made in stepping from $p, \neg p$ to $q$ only makes sense if that ' $\neg$ ' is a genuine negation. If we weaken our interpretation to make ' $\neg$ ' not mean not but something weaker ("possibly not," say) then $p, \neg p$ are not inconsistent: we have merely the appearance of inconsistency, not its substance.

What goes for pluralism about relevant (or paraconsistent) and classical consequence also goes for the contrast between classical and constructive validity [9]. There are a number of different ways to be a constructive mathematicianbut there is a significant tradition in constructive mathematics, according to which constructive mathematics is the study in which we constructively reason about everyday mathematical entities. There are no special or distinctive 'constructive real numbers' - you constructively prove things about the real numbers. Proponents of this view are Errett Bishop [3] and Fred Richman [13, 14], $!^{2}$ On this view, when we use the weaker classical criteria for evaluating arguments, the argument from $\neg \neg p$ to $p$ is valid: it never steps from truth to untruth. However, the very same argument can fail to be constructively valid. When we fault the argument from $\neg \neg p$ to $p$ on these constructive grounds it is not that $\neg \neg p$ can be true while $p$ fails to be true 3 -it is that we can have a construction of

\footnotetext{
${ }^{1}$ For an exposition of this kind of view, see "Negation in Relevant Logics" [8].

${ }^{2}$ Douglas Bridges and Fred Richman's Varieties of Constructive Mathematics [5] contains a helpful outline of the different schools of constructive mathematics. Intuitionism, Russian Constructivism and Recursive Analysis are all theories which can be thought of as either providing different concflicting theories of real numbers, or theories governing distinctive kinds of numbers-such as recursive reals. Bishop-style constructive mathematics is explicitly constructively reasoning about mathematical objects-objects about which we may (if we wish) reason classically, too.

${ }^{3} \mathrm{~A}$ referee points out that this is something to which all friends of intuitionist logic can agree.
} 


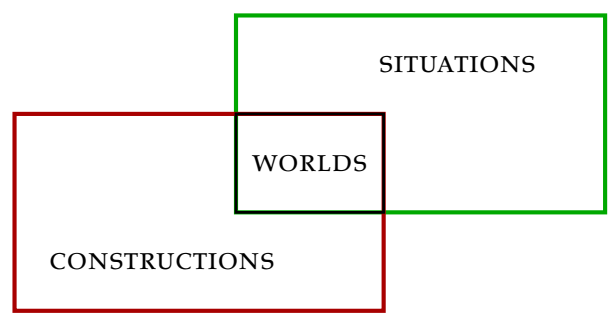

Figure 1: THREE CLASSES OF CASES

$\neg \neg p$ which isn't thereby a construction of $p$. Again here, it is important that ' $\neg$ ' be negation. If it is something stronger than negation (say, "provably not the case that" instead of "it is not the case that"), then we do not take the one and the same argument to be valid classically and invalid constructively. But this is one way to understand constructive mathematics: it is an approach to mathematics where some theses which are classically derivable are not constructively derivable.

These examples underlie the view presented in Logical Pluralism [2] 4 The view developed there is motivated specific examples of concrete arguments which we take to be valid in one sense and invalid in another. That is one characteristic feature of the kind of pluralism developed in Logical Pluralism [2].

The next characteristic feature of the view is the analysis of logical consequence as preservation of truth in cases. Different consequence relations arise from different ways to carve out the class of cases used in characterising those consequence relations. The result is the sort of picture in which we have one language in which arguments are expressed, and at least three different consequence relations on that language, found by choosing different classes of cases to define logical consequence. Here, classical consequence is found by quantifying over worlds, intuitionistic consequence is found by quantifying over constructions (some of which are the worlds), which can allow for gaps, which need not decide between $p$ and $\neg p$ for each $p$, and a paraconsistent consequence relation is found by quantifying over what we will call situations, which allow for gluts,

Since $\vdash \neg(\neg \neg p \wedge \neg p)$, if we have a transparent theory of truth, the intuitionist can endorse $\neg(\mathrm{T}\langle\neg \neg p\rangle \wedge \neg \mathrm{T}\langle\mathrm{p}\rangle)$. It is never the case that $\neg \neg p$ is true while $p$ is not. This is a particularly sharp way of making the point.

${ }^{4}$ There are more motivations than this. One motivation we do not discuss much is the treatment of incompleteness and indeterminacy. We can take it that the tautology $p \vee \neg p$ is not settled by each and every thing, but nonetheless take it that $p \vee \neg p$ is of necessity made true by something. Is $p \vee \neg p$ a logical truth? Depends on the criterion. Not every restricted part of the world makes each instance of $p \vee \neg p$ true for it might not involve the feature which makes $p$ true or rules it out. However, every world involves such a feature [6 [1]. (Of course, this phenomenon is not restricted to claims involving negation. We might think, for example, that the tautology $E ! a \supset a=a$-if $a$ exists then it is self identical—is made true, if a exists, by parts of the world featuring a.) 
points at which $p$ and $\neg p$ both hold 5

This picture relies on a model-theoretic truth-preservation account of logical consequence. A lacuna in the book is that we do not say much at all about proof theory. While the difference between classical and constructive logic can be well understood by way of Kripke or Beth models, it does not need to be understood in this way. Instead, we can understand the difference between classical logic and intuitionistic logic in terms of proofs. If we think of intuitionistic logic and classical logic in terms of proofs, do we end up with the same view of the relation between different logics, or do we get a different kind of logical pluralism? That is the topic for this paper.

\section{》 INTUITIONIST, DUAL INTUITIONIST, AND CLASSICAL NEGATIONS}

To keep things specific, we will consider inference rules involving negation. Consider the rules from Gentzen's sequent calculus for classical logic. The classICAL rules for negation are as follows:

$$
\frac{X \vdash A, Y}{X, \neg A \vdash Y}[\neg L] \quad \frac{X, A \vdash Y}{X \vdash \neg A, Y}[\neg R]
$$

These rules manipulate sequents in which finite collections of formulas appear on the left- and the right-hand sides of the separating turnstile. If we wish to understand a derivable sequent $\mathrm{X} \vdash \mathrm{Y}$ as constraining boolean evaluations, we can think of it negatively (no evaluation takes each element of $X$ to be true and each element of $Y$ to false) or positively (every evaluation which takes every element of $X$ to be true takes some element of $Y$ to be true; or equivalently, every evaluation which takes every element of $Y$ to be false takes some element of $X$ to be false). The connective rules such as these rules for negation are supplemented with structural rules which appeal to no particular kind of formula. First, we have an identity rule, such as this:

$$
X, A \vdash A, Y
$$

and a rule to $C u t$ out intermediate formulas:

$$
\frac{X \vdash Y, A \quad A, X \vdash Y}{X \vdash Y}
$$

The rules for negation allow us to derive classically valid negation principles in an elegant, symmetrical manner:

$$
\frac{\frac{p \vdash p}{p, \neg p \vdash}[\neg L]}{p \vdash \neg \neg p}[\neg R] \quad \frac{p \vdash p}{\vdash \neg p, p}[\neg R]
$$

5'Situations' in Logical Pluralism [2] allow for truth value gaps and truth value gluts. Here we will consider only truth value gluts in these situations. They are closer, on this conception, to the inconsistent worlds of "Ways Things Can't Be" [7], which are collections of consistent and complete worlds considered conjunctively. 
If we supplement the rules for negation with standard rules for conjunction and disjunction

$$
\begin{array}{ll}
\frac{X, A \vdash Y}{X, A \wedge B \vdash Y}\left[\wedge L_{1}\right] & \frac{X, B \vdash Y}{X, A \wedge B \vdash Y}\left[\wedge L_{2}\right] \\
\frac{X \vdash A, Y}{X \vdash A \vee B, Y}\left[\vee R_{1}\right] & \frac{X \vdash B, Y}{X \vdash A \vee B, Y}\left[\vee R_{2}\right]
\end{array}
$$

we may derive the following sequents

$$
\begin{array}{cc}
\frac{p \vdash p}{p, \neg p \vdash}[\neg L] & \frac{p \vdash p}{\vdash p, \neg p}[\neg R] \\
\frac{p \wedge \neg p, \neg p \vdash}{p \wedge \neg p \vdash}\left[\wedge L_{1}\right] & \frac{\vdash p \vee \neg p, \neg p}{\vdash p \vee}\left[\vee R_{1}\right] \\
\frac{\left.\vdash p R_{2}\right]}{}[p / p
\end{array}
$$

the first of which tells us that $p \wedge \neg p$ is a contradiction (never true, always false), and the second, that $p \vee \neg p$ is a tautology (always true, never false).

One of Gentzen's great insights in proof theory was that these rules also provide us with rules for negation if we are to reason using the strictures of intuitionist logic. If we take the same rules but be careful to use only proofs in which there is at most one formula on the right hand side of a sequent, the instances of the rules we will use are these, the intuitionist instances of the rules for negation.

$$
\frac{\mathrm{X} \vdash \mathrm{A}}{\mathrm{X}, \neg \mathrm{A} \vdash}[\neg L] \quad \frac{\mathrm{X}, \mathrm{A} \vdash}{\mathrm{X} \vdash \neg \mathrm{A}}[\neg R]
$$

and similarly, we must use only restricted forms of the other rules, such as the rules for conjunction and disjunction:

$$
\begin{array}{cl}
\frac{X, A \vdash C}{X, A \wedge B \vdash C}\left[\wedge L_{1}\right] & \frac{X, B \vdash C}{X, A \wedge B \vdash C}\left[\wedge L_{2}\right] \\
\frac{X \vdash A}{X \vdash A \vee B}\left[\vee R_{1}\right] & \frac{X \vdash B}{X \vdash A \vee B}\left[\wedge R_{2}\right]
\end{array}
$$

We can impose exactly the same kind of restriction, but on the left rather than on the right. If we allow only derivations in which there is at most one formula on the left, the remaining instances of the rules for negation are these.

$$
\frac{\vdash \mathrm{A}, \mathrm{Y}}{\neg \mathrm{A} \vdash \mathrm{Y}}[\neg L] \quad \frac{\mathrm{A} \vdash \mathrm{Y}}{\vdash \neg \mathrm{A}, \mathrm{Y}}[\neg R]
$$

The conjunction or disjunction rules we have mentioned are similarly restricted:

$$
\frac{A \vdash Y}{A \wedge B \vdash Y}\left[\wedge L_{1}\right] \quad \frac{B \vdash Y}{A \wedge B \vdash Y}\left[\wedge L_{2}\right]
$$




$$
\frac{\mathrm{C} \vdash \mathrm{A}, \mathrm{Y}}{\mathrm{C} \vdash \mathrm{A} \vee \mathrm{B}, \mathrm{Y}}\left[\vee R_{1}\right] \quad \frac{\mathrm{C} \vdash \mathrm{B}, \mathrm{Y}}{\mathrm{C} \vdash \mathrm{A} \vee \mathrm{B}, \mathrm{Y}}\left[\vee R_{1}\right]
$$

We will call these the dual-intuitionist instances of the negation rules. In general, we have the following three kinds of derivations.

- A classical derivation is a tree of sequents, whose leaves are the axioms, and whose transitions are instances of the rules: the connective rules or the structural rules.

- An intuitionist derivation is a classical derivation in which the negation steps satisfy the constraint: at most one formula present on the RHS.

- A dual-intuitionist derivation is a classical derivation in which the negation steps satisfy the constraint: at most one formula present on the LHS.

So, intuitionist and dual-intuitionist derivations are classical derivations with extra restrictions. Corresponding to these restrictions are different virtues.

- A derivation can have the virtue of being classically valid.

- It can also have the virtue of being intuitionistically valid.

- It can also have the virtue of being dual-intuitionistically valid.

- Some derivations have all three virtues.

This way of understanding the relationship between the three logics-classical, intuitionist and dual intuitionist logic-coheres with the motivations with which we began. The classical account of validity takes $p \vee \neg p$ to be derivable, for it has a derivation which meets the (weak) classical criteria. This derivation also meets the dual-intuitionistic criterion: there is never more than one formula on the LHS, so the very same statement, $p \vee \neg p$, is provable in dual-intuitionist logic. However, it has no derivation in which there is at most one formula on the RHS: $p \vee \neg p$ is not intuitionistically provable. The one and the same statement, $p \vee \neg p$, is both a classical and a dual-intuitionist tautology, but it is not an intuitionist tautology. The picture, then, has three classes of derivations, the comprehensive class of classical derivations, and two overlapping subclasses, of single-right (intuitionistic) and single-left (dual-intuitionistic) derivations.

On this picture, we have one language, and three logical consequence relations on that language, by having one family of derivations and various different criteria which may be applied to those derivations ${ }^{6}$ Given this picture, what can we say about models for these logics? Does this give a distinct perspective on the cases which we may use to characterise validity? After all, the soundness and completeness results for each of these logics may be interpreted in at least

\footnotetext{
${ }^{6}$ Of course, there are examples of arguments which are classically valid and neither intuitionistically or dual intuitionistically valid. A simple example is the argument from $p \wedge \neg p$ to $q \vee \neg q$.
} 


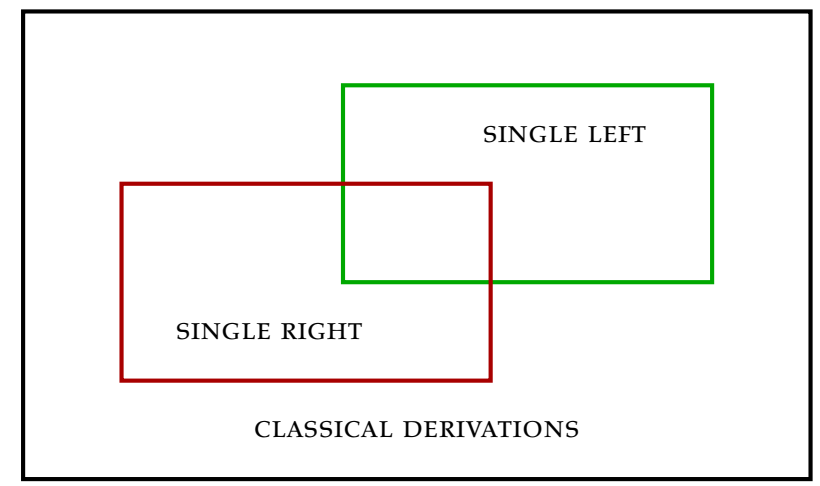

Figure 2: THREE CLASSES OF DERIVATIONS

two different ways. We can think of the model-theoretic account of validity as primary, and think of soundness and completeness of the proof theory as a criterion for the acceptability of that proof-theoretic account. But we can think of soundness and completeness in reverse: perhaps the proof-theoretic account of validity is primary, and soundness and completeness for the model theory is a criterion for the acceptability for that model-theoretic analysis. If we think of models in this way: as justified and motivated by way of the proofs, then perhaps this will give us a different perspective on how to understand cases in a model. So, it is to this that we will turn.

> THREE NEGATIONS IN ONE LOGIC?

The reason for paying attention here is a problem for the view that we have three different consequence relations in one vocabulary. If we think of three different accounts of a model theory for our three logics, then it might appear that we have a compelling case to conclude not that we have three different logics of the one notion of negation (which I have been defending thus far), but that we genuinely have three different concepts of negation. Here is how the problem arises. When we think of the truth conditions for negation, we think of something like this:

$$
x \Vdash \neg A \text { iff } x \Vdash A
$$

A negated statement holds at a point iff the statement which is negated fails to hold at that point. Then, if we have some class of these points at which statements are evaluated, we can define logical consequence (relative to that model) in the usual manner:

$\mathrm{X} \vdash_{\mathrm{K}} \mathrm{Y}$ iff there's no $\mathrm{x}$ where $\mathrm{x} \Vdash A$ for each $A \in \mathrm{X}$ and $\mathrm{x} \| \mathrm{B}$ for each $\mathrm{B} \in \mathrm{Y}$ and the result is that $\mathrm{X} \vdash \mathrm{Y}$ is classically derivable if and only if $\mathrm{X} \vdash_{\mathrm{K}} \mathrm{Y}$ holds in every model. 
For intuitionist logic the story is slightly more complicated. This clause for negation will not do. Rather, points in a model (whether it is a Kripke model or a Beth model will not matter for our purposes) come ordered by a partial order $\leqslant$, such that

$$
\text { Now if } x \Vdash A \text { and } x \leqslant x^{\prime} \text {, then } x^{\prime} \Vdash A \text { too. }
$$

This is well motivated by the idea of taking points as constructions, and we notice that constructions can be incomplete (construct neither $A$ nor $\neg A$ ) and be expanded to construct more. This conception of a construction means that the classical clause for negation is not appropriate (if $x$ constructs neither $A$ nor $\neg A$ then the 'if' part of ' $x \Vdash \neg A$ iff $x \| A^{\prime}$ ' fails), and must be replaced by a different condition. The usual condition in these models is as follows:

$$
x \Vdash \neg A \text { iff } x^{\prime} \| \forall A \text { for every } x^{\prime} \geqslant x .
$$

Here, a construction validates a negation iff every way of extending it is unable to construct the formula negated. In other words, there is some way of extending $x$ to validate $A$ if and only if $x$ does not construct $\neg A$. Using this different notion of a model (a family of points, ordered by $\leqslant$ in which negation is evaluated in that way), we may define intuitionist consequence in exactly the same way as classical consequence.

$X \vdash_{\text {J }} \mathrm{Y}$ iff there's no $\mathrm{x}$ where $\mathrm{x} \Vdash A$ for each $A \in X$ and $x \| B$ for each $B \in Y$

If we have a model consisting of two points $\mathrm{a}$ and $\mathrm{b}$ where $\mathrm{a}<\mathrm{b}$ and $\mathrm{a} \| \mathrm{p}$ but $\mathrm{b} \Vdash \mathrm{p}$ then $\mathrm{a} \forall \neg p$ and $\mathrm{a} \Vdash \neg \neg p$. It follows that $\neg \neg p \nvdash$ J $p$ since at point $\mathrm{a}$ in this model, $\neg \neg p$ holds but $p$ doesn't.

For dual-intuitionist logic, you do exactly the same thing, except we reverse the order and replace universal quantifiers by existential quantifiers.

$$
x \Vdash \neg A \text { iff } x^{\prime} \| A \text { for some } x^{\prime} \leqslant x .
$$

then we have

$$
\text { Now if } x \Vdash A \text { and } x \leqslant x^{\prime} \text {, then } x^{\prime} \Vdash A \text { too. }
$$

and we can define dual-intuitionist consequence in exactly the same manner, but in these models instead of classical or intuitionist models.

$X \vdash_{D J} Y$ iff there's no $x$ where $x \Vdash A$ for each $A \in X$ and $x \| B$ for each $B \in Y$

If we have a model consisting of two points $\mathrm{c}$ and $\mathrm{d}$ where $\mathrm{d}<\mathrm{c}$ and $\mathrm{c} \Vdash \mathrm{p}$ but $\mathrm{d} \Downarrow \mathrm{p}$ then $\mathrm{c} \Vdash \neg p$ since $\mathrm{d}<\mathrm{c}$ and $\mathrm{d} \forall p$. However, $\mathrm{c} \Vdash \mathrm{p}$ too (so $\mathrm{c}$ is inconsistent about $\mathrm{p}$ ) and furthermore, $\mathrm{c} \Downarrow \neg \neg \mathrm{p}$ since $\mathrm{c} \Vdash \neg p$ and also, $\mathrm{d} \Vdash \neg p$. So, this model has a point (c) at which $p$ holds but $\neg \neg p$ fails. We have shown that $p \nvdash$ D J $\neg \neg p$. 
Notice that in these models the points which are over-determined, by being inconsistent between $p$ and $\neg p$. Now points can be thinned out into more discriminating points which over-determine fewer statements. The heredity condition plays exactly the same role, but now $c \leqslant d$ means that $d$ is at least as inconsistent as $\mathrm{c}$ is, while $\mathrm{c}$ may thin out some of the inconsistencies in $\mathrm{d}$.

Now, given this understanding of negation in classical, intuitionist and dualintuitionist models, we should address another question:

- Given a frame $\langle\mathrm{P}, \leqslant\rangle$, a satsfaction relation $\Vdash$ and a point $\mathrm{x}$ in that frame how can we check for whether or not $x \Vdash \neg A$ ?

It seems we have at least three different answers. One approach is to say that we have three negations, not one. In $\langle\mathrm{P}, \leqslant\rangle$ we could have

$$
\begin{aligned}
& -x \Vdash-A \text { iff } x \| A . \\
& -x \Vdash \smile A \text { iff } x^{\prime} \forall A \text { for each } x^{\prime} \geqslant x . \\
& -x \Vdash \frown A \text { iff } x^{\prime} \forall A \text { for some } x^{\prime} \leqslant x .
\end{aligned}
$$

As we have seen, evaluating negation using the classical clause, the intuitionist clause, and the dual-intuitionist clause each result in very different logical behaviours. What are we to make of the three different answers to this question? It seems that allowing for three different answers points to a kind of pluralism in which the logical notions have bifurcated (or 'trifurcated'). Can this happen?

No, it cannot. At least, it cannot 'trifurcate' in this way. There is no frame in which all three negations coexist as propositional operators on the same class of propositions, giving the distinct classical, intuitionist and dual-intuitionist properties. If a frame is non-trivial-if there are points $a$ and $b$ where $a<b$, then we may set $a \Vdash p$ and $b \Vdash p$. If we were to have a negation ' ${ }^{\prime}$ ' evaluated by way of the classical clause on this frame, then $a \Vdash-p$ and $b \Vdash-p$. That is, even though $b$ extends $a$, it is no longer the case that more statements are true at $b$ than were true at $a$. The heredity of information from point to point breaks down 7 So we cannot have three different negations on the one frame without breaking heredity. We could however, have the negations of $\smile$ and $\frown$ on the one frame, without breaking heredity. Both negation clauses produce hereditary propositions, preserved up and down the ordering. If we have a frame on the two points $\mathrm{a}$ and $\mathrm{b}$ where $\mathrm{a}<\mathrm{b}$ and $\mathrm{a} \| p$ but $\mathrm{b} \Vdash \mathrm{p}$ as before, then if we evaluate $\smile$ and $\frown$ on this frame, we have the following results:

\footnotetext{
${ }^{7}$ If we allow for two kinds of propositions, one, genuinely hereditary, and another, not heredity, we would have a way for - to 'coexist' after a manner of speaking with $\smile$, following the intuitionist clause, and $\frown$, following the dual-intuitionist clause. However, the information expressed by means of the classical clause for ' - ' is no longer hereditary, and it is not suitable for use in any context in which this hereditary information is necessary.
} 


\begin{tabular}{lccccc}
\hline & $p$ & $\smile p$ & $\smile \smile p$ & $\frown p$ & $\frown \frown p$ \\
\hline$a$ & - & - & + & + & - \\
$b$ & + & - & + & + & - \\
\hline
\end{tabular}

The two negations are very different (the single negation of $p$ for one is, in this model, equivalent to the double negation of $p$ for the other!), but they can, after a fact, coexist. This points to the possibility of a kind of 'Carnapian' pluralism [10] in which we are bilingual speaking two different languages with two different negation connectives. On this picture, there's one logic, encompassing statements expressible in two different vocabularies ${ }^{8}$ This pluralism is very different from the pluralism of the book Logical Pluralism. It is certainly a coherent approach. What can we say about this kind of pluralism, given the proof-theoretic approach of this paper? We would like to have some principle by which we can explain why multiple differing interpretations of negation on the one frame are not appropriate. But what could that be?

\section{OR ONE NEGATION IN THREE LOGICS?}

If we start with the proof-theory, a point in a model is to be understood as a site for a counterexample of an invalid argument. If, in our logic, we cannot derive $B$ from $A$ then there is some point (in some model) at which $A$ holds and B fails. As has been shown elsewhere [12], this can motivate a very general connection between sequent calculi and frames for different logics. The slogan is that we can treat a point in a model as an idealised underivable sequent. If we cannot derive $A$ from $B$ then the pair $[A ; B]$ must have some counterexample. We can find a point where $A$ is among the claims that are true, and $B$ is among the claims that are false. But points never make just one formula true (or just one formula false). They evaluate the formulas en masse and each point determines a partition of the language $[X ; Y]$ into the statements evaluated as true $(X)$ and those evaluated as false $(y)$. The crucial feature for such partitions is that they are never bridged by a derivable sequent. We must never have $X \subseteq X$ and $Y \subseteq Y$ where $\mathrm{X} \vdash \mathrm{Y}$.

We call a pair $[X ; Y]$ of sets of formulas a COUNTEREXAMPLE whenever $X^{\prime} \not Y^{\prime}$ for any finite $X^{\prime}, Y^{\prime}$ where $X^{\prime} \subseteq X$ and $Y^{\prime} \subseteq Y$.

Note that if $X$ and $Y$ are finite, $[X ; Y]$ is a counterexample just when we do not have a derivation for $X \vdash Y$. If either $X$ or $Y$ are infinite sets, then strictly speaking, they cannot feature in a sequent, hence the form of the definition.

Now, just as a point in a space can be understood as a limit of a process of sharpening a region, we can will define a point for one of our models to be a similarly sharpened counterexample.

\footnotetext{
${ }^{8}$ And once one is bilingual, we can consider mixed statements. We have, for example, $\smile A \vdash$ $\frown A$, since if $x \Vdash \smile A$ then $x \| A$ and hence, $x \Vdash \frown A$.
} 
A POINT is a counterexample where $[X ; Y]$ is a partition of the entire language. Every formula is either in $X$ or it is in $y$.

ғаст: Every counterexample $[X ; Y]$ may be refined into a point $[X ; Y]$ where $X \subseteq$ $X$ and $Y \subseteq y$.

Proof: The process is straightforward. Consider the class of all counterexamples. Order them by the component-wise subset relation: $[X ; Y] \sqsubseteq\left[X^{\prime} ; Y^{\prime}\right]$ when $X \subseteq$ $X^{\prime}$ and $Y \subseteq Y^{\prime}$. For any counterexample $[X ; Y]$ and formula $A$, either $[X, A ; Y]$ or $[X ; A, Y]$ is a counterexample, too. (This follows from the $C u t$ rule.) So, given a counterexample $[X ; Y]$ choose a maximal $\sqsubseteq$-directed class of counterexamples, to which $[\mathrm{X} ; \mathrm{Y}]$ belongs. This has a maximum, which is a partition of the language.

This construction works whether we pay attention to classical derivations of sequents, if we restrict our attention to intuitionist derivations or to dualintuitionist derivations. An intuitionist partition is a pair $[X ; Y]$ where $X^{\prime} \vdash C$ has no intuitionist derivation for any finite $X^{\prime} \subseteq X$ and $C \in Y$. The dual definition works for dual-intuitionist derivations, as in either case, partitions may be extended by points. For example, if $[X ; Y]$ is a dual-intuitionist partition (so $C \vdash Y^{\prime}$ is underivable whenever $C \in X$ and $Y^{\prime} \subseteq Y$ ), then either $[X, A ; Y]$ or $[X ; A, Y]$ is a dual-intuitionist partition too. Here's why: suppose $[X, A ; Y]$ and $[X ; A, Y]$ are not partitions. If $[X, A ; Y]$ is not a partition, then either $C \vdash Y^{\prime}$ for some $C \in X$ and $Y^{\prime} \subseteq Y$, and hence $[X ; Y]$ is not a partition, contrary to what is assumed, or on the other hand, $A \vdash Y^{\prime}$ for some subset $Y^{\prime}$ of $Y$. Now $[X ; A, Y]$ is not a partition, so there is some $C^{\prime} \in X$ where $C^{\prime} \vdash A, Y^{\prime \prime}$ for some $Y^{\prime \prime} \subseteq Y$. Now, we have $C^{\prime} \vdash A, Y^{\prime \prime}$ and $A \vdash Y^{\prime}$, and so, by $C u t$, we have $C^{\prime} \vdash Y^{\prime}, Y^{\prime \prime}$ where $Y^{\prime}, Y^{\prime \prime} \subseteq Y$ and $C^{\prime} \in X$. This tells us that $[X ; Y]$ is not a partition, contrary to what was assumed. (The reasoning is exactly dual in the case of intuitionist logic. 9

So, since sequents are invalid in different ways, there are different kinds of points. Some points are idealised classically invalid sequents, while others are idealised intuitionistically invalid sequents, or dual-intuitionistically invalid sequents.

- We will call classical points, wORLDS

- Intuitionist points, CONSTRUCTIONS

- Dual Intuitionist points, SITUATIONS

9I have discussed this construction of points from invalid sequents elsewhere [12]. In that paper, I show that if we allow multiple conclusion sequents for intuitionist logic and restrict the rules for the conditional and negation, but not conjunction or disjunction, the resulting structure of points is noticeably different to what we have here. As we will see, the structure of points here forms a Beth model for intuitionist logic, in which a disjunction can hold at a point without either disjunct holding at that point. The construction applied to multiple conclusion sequents forms a Kripke model. The distinction between Kripke and Beth models for intuitionist logic will play no role in what follows. 
In line with our previous vocabulary. A point describes how things might be true and other things might be false. We will say that $A$ is true at $[X ; Y]$ iff $A \in X$

ғаст: $A \wedge B$ is true at $[X ; Y]$ iff $A$ and $B$ are both true at $[X ; Y]$-if $[X ; Y]$ is either a world or a construction.

Proof: Suppose $A \wedge B$ is in $X$. Then since $A \wedge B \vdash A$, and $A \wedge B \vdash B$, neither $A$ nor $B$ are in $y$. So, since points are partitions, $A$ and $B$ are both in $X$. Conversely, suppose $A$ and $B$ are in $X$. Then since $A, B \vdash A \wedge B$ (this is where the argument fails for situations: we need two formulas on the left of the sequent), we cannot have $A \wedge B \in Y$. So, since points are partitions, $A \wedge B$ is in $X$.

For disjunction, we may follow the same sort of reasoning, but you dualise. The standard clause will work for worlds and for situations, but not for constructions $\stackrel{10}{10}$

Negation is more interesting. For worlds, we have the following fact:

ғаст: If $[X ; Y]$ is a world, then $\neg A$ is true at $[X ; Y]$ iff $A$ is not true there.

Proof: Suppose $\neg A$ is in $X$. Then since $A, \neg A \vdash, A$ isn't in $X$. So, since points are partitions, $A$ is in $y$. Suppose $A$ is in $y$. Since $\vdash A, \neg A$, we cannot have $\neg A$ in $y$. So, since points are partitions, $\neg A$ is in $X$.

This reasoning relies on having $A, \neg A \vdash$ and $\vdash A, \neg A$, so it works for $\vdash_{K}$, but neither $\vdash_{\mathrm{J}}$ nor $\vdash_{\mathrm{DJ}}$.

For constructions, the reasoning is more subtle:

FACT: If $[X ; Y]$ is a construction, then $\neg A$ is true at $[X ; Y]$ iff $A$ is not true at any construction $\left[X^{\prime} ; y^{\prime}\right]$ where $X \subseteq X^{\prime}$.

Proof: Suppose $\neg A$ is in $X$. Then since $A, \neg A \vdash, A$ isn't in $X$ and $A$ isn't in $X^{\prime}$ for any construction $\left[X^{\prime} ; y^{\prime}\right]$ where $X \subseteq X^{\prime}$. So, in each of these cases, $A$ is in $y^{\prime}$.

Suppose $\neg A$ is not in $X$. So, since points are partitions, $\neg A$ in $Y$. Then, since $X \forall \neg A$ for any $X \subseteq X$, we must have $X, A \forall$ for any $X \subseteq X$ too. So, it follows that there is a point extending $[X, A ;]$. This is a point $\left[X^{\prime} ; \mathcal{Y}^{\prime}\right]$ at which $A$ is true. Contraposing, if $A$ is not true at $\left[X^{\prime} ; Y^{\prime}\right]$ for each $X^{\prime} \supseteq X$, then $\neg A$ is in $X$.

For 'situations' we have a dual fact. We have:

ғаст: If $[X ; Y]$ is dual-intuitionisically invalid, then $\neg A$ is true at $[X ; Y]$ iff $A$ is not true at some point $\left[X^{\prime} ; y^{\prime}\right]$ where $y \subseteq y^{\prime}$.

Proof: dualise.

So, these three kinds of points: worlds, constructions and situations, deserve the name. Truth of a negation at a world satisfies the classical rules for negation.

\footnotetext{
${ }^{10}$ What clause will work in constructions? The bar condition from Beth Semantics [12].
} 


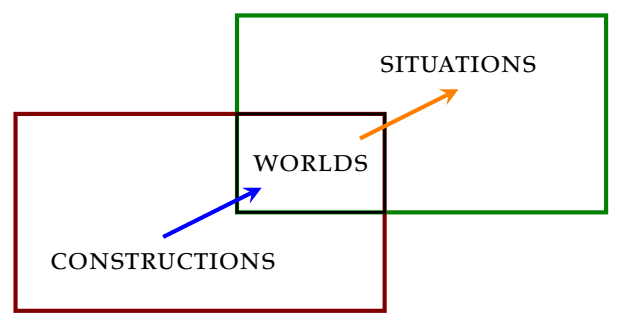

Figure 3: THREE CLASSES OF CASES-WITH ADDED ORDERINGS

Truth of a negation at a construction satisfies the intuitionist rules for negation, where the ordering is the subset relation on the left of the partition. Truth of a negation at a situation satisfies the dual-intuitionist rules for negation, where the ordering is the subset relation in the right of the partition. So, negation here is the one concept, and the variation in clauses results not from the difference between negations at each point, but rather, in the points themselves ${ }^{11}$ Negation is a single item, and the difference between worlds, constructions and situations governs the different behaviour of negation at those three different sites.

This motivates our original picture of worlds, constructions and situations, from a proof theoretical perspective. Now we not only have three different kinds of points at which formulas are evaluated. Now, we have an explanation of why the different logical properties of negation are observed at these points, given the underlying unity. On this account, negation is what it is, because of the rules $[\neg L / R]$. Worlds are idealised sequents with no classical derivation. Constructions are also idealised sequents, but they may be bridged by a classical derivation, so long as a stricter intuitionist derivation cannot bridge the gulf between the left and the right. Similarly, situations are idealised sequents, where no dual-intuitionist derivation can bridge that gulf.

Furthermore, the relation in the point semantics for these logics is illuminated. Despite the fact that we have hereditary conditions on both the intuitionist preservation relation and its dual-intuitionist sibling, we see that these are not the same relation. For constructions, it is the subset ordering on the left hand side, and it ranges over constructions (which include worlds). Worlds are endpoints in this ordering. For each world, there is no world (or no point at all)

\footnotetext{
${ }^{11}$ We have not provided a uniform truth condition for negation over a frame with points which are constructions, worlds and situations. A referee pointed out that this might be thought to indicate that negation still not truly one thing but three, in this model, because at worlds it is evaluated using the boolean clause, at constructions, a universal forward-looking clause, and at situations, a particular backward-looking clause. To think that is to think that the area of a plane figure is not 'one thing' because for circles it can be calculated in one way, ellipses another, squares another, and so on. The fact that the techniques agree where they overlap should help make the point that there are not three separate notions, any more than that the areas of a circle, a square or an ellipse are a different kinds of area.
} 
further along in this ordering. Worlds are consistent and complete with respect to negation, and constructions are consistent, but they are not complete. For situations, the result is reversed-worlds are the initial points in the ordering, and the other situations, further along, allow for inconsistency, but maintain compeleteness. This relation is between situations. No construction is earlier in an ordering to any situation (except when both are worlds-and in fact, only when both are the same world). So, it is not really as if the same relation of preservation of truth leads us from constructions, through worlds, to situations.

Proof theory has given us an understanding of model theory. On this view, we have learned the following three lessons:

- Negation is the one thing.

- It works differently at different places.

- This different behaviour is illuminated by different strictures on proofs.

A focus on proofs does not lead away from the kind of logical pluralism we have discussed. It motivates that pluralism further, and gives us new ways to understand it.

\section{REFERENCES}

[1] JC Beall and greg Restall. "Logical Pluralism". Australasian Journal of Philosophy, 78:475-493, 200o. http://consequently.org/writing/pluralism

[2] JC Beall and greg restall. Logical Pluralism Oxford University Press, Oxford, 2006.

[3] ERrett Bishop. Foundations of Constructive Analysis. McGraw-Hill, 1967. Out of print. A revised and extended version of this volume has appeared [4].

[4] errett bishop AND douglas bridges. Constructive Analysis. Springer-Verlag, 1985.

[5] DOUgLAS S. BRIDGES AND FRED RICHMAN. Varieties of constructive mathematics, volume 97 of London Mathematical Society Lecture Notes. Cambridge Univ Press, 1987.

[6] GReg Restall. "Truthmakers, Entailment and Necessity". Australasian Journal of Philosophy, 74:331-340, 1996.

[7] Greg Restall. "Ways Things Can't Be". Notre Dame Journal of Formal Logic, $38(4): 583-596,1997$.

[8] GREg RESTALl. "Negation in Relevant Logics: How I Stopped Worrying and Learned to Love the Routley Star". In DOv GABBAY AND HEINRICH WANSING, editors, What is Negation?, volume 13 of Applied Logic Series, pages 53-76. Kluwer Academic Publishers, 1999.

[9] GReg Restall. "Constructive Logic, Truth and Warranted Assertibility". Philosophical Quarterly, 51:474-483, 2001. http://consequently.org/writing/conlogtr/

[10] GReg Restall. "Carnap's Tolerance, Meaning and Logical Pluralism". The Journal of Philosophy, 99:426-443, 2002. http://consequently.org/writing/carnap/

[II] GReg Restall. "Lukasiewicz, Supervaluations and the Future". Logic and Philosophy of Science, 3(I):I-10, 2005.

[12] GReg Restall. "Truth Values and Proof Theory". Studia Logica, 92(2):241-264, 2009. http://consequently.org/writing/tvpt/

[13] FRED RICHMAN. "Intuitionism as Generalization". Philosophia Mathematica, 5:124-128, 1990. 
[14] FRED RICHMAN. "Interview with a constructive mathematician". Modern Logic, 6:247-271, 1996. 